\title{
Decompositions of Complete Multigraphs into Cyclic Designs
}

\author{
Mowafaq Alqadri ${ }^{\#}$, Haslinda Ibrahim ${ }^{\#}$, Sharmila Karim ${ }^{\#}$ \\ \# Department of Mathematics, School of Quantitive Sciences, College of Arts and Sciences, Universiti Utara Malaysia, Kedah, Malaysia \\ E-mail: moufaqq@yahoo.com,linda@uum.edu.my,mila@uum.edu.my
}

\begin{abstract}
Let $v$ and $\lambda$ be positive integer, $\lambda K_{V}$ denote a complete multigraph. A decomposition of a graph $G$ is a set of subgraphs of $G$ whose edge sets partition the edge set of $G$. The aim of this paper, is to decompose a complete multigraph $4 K_{v}$ into cyclic $(v-1$ ) cycle system according to specified conditions. As the main consequence, construction of decomposition of $8 K_{v}$ into cyclic Hamiltonian wheel system, where $v \equiv 2(\bmod 4)$, is also given. The difference set method is used to construct the desired designs.
\end{abstract}

Keywords - Cyclic design; Hamiltonian cycle, Near four factor, Wheel graph.

\section{INTRODUCTION}

Throughout this paper, all graphs consider finite and undirected. A complete graph of order v denotes by K_v. An $(\mathrm{G}, \mathrm{Y})$-design is a decomposition of the graph $\mathrm{G}$ into subgraphs belonging to an assigned multiset $\mathrm{Y}$.

An m-cycle, written $C_{-} m=\left(c_{-} 0, \cdots, c_{-}(m-1)\right)$, consists of $\mathrm{m}$ distinct vertices $\left\{\mathrm{c}_{-} 0, \mathrm{c}_{-} 1, \cdots, \mathrm{c}_{-}(\mathrm{m}-1)\right\}$ and $\mathrm{m}$ edges $\{\mathrm{c}$ i c_(i+1) $\}, 0 \leqslant \mathrm{i} \leqslant \mathrm{m}-2$ and $\mathrm{c}_{-} 0$ c_(m-1) and m-cycle of a graph $G$ is called Hamiltonian when its vertices passes through all the vertex set of G. An m-path, written [c_0, $\left.\cdots, c \_(m-1)\right]$, consists of $m$ distinct vertices $\left\{c \_0, c \_1, \cdots, c \_(m-1)\right\}$ and $m-1$ edges $\left\{c \_i c \_(i+1)\right\}, 0 \leqslant i \leqslant$ $\mathrm{m}-2$. An m-cycle system of a graph $\mathrm{G}$ is $(\mathrm{G}, \mathrm{C})$-design where $\mathrm{C}$ is a collection of $\mathrm{m}$-cycles. If $\mathrm{G}=\mathrm{K} \_\mathrm{v}$ then such $\mathrm{m}$-cycle system is called m-cycle system of order $\mathrm{v}$ and is also said a simple when its cycles are all distinct.

An automorphism group on $(\mathrm{G}, \mathrm{Y})$-design is a bijections on $\mathrm{V}(\mathrm{G})$ fixed $\mathrm{Y}$. An $(\mathrm{G}, \mathrm{Y})$-design is a cyclic if it admit automorphism group acting regularly on $V(G)$ [1]. For a cyclic $(\mathrm{G}, \mathrm{Y})$-design, we can assume that $\mathrm{V}(\mathrm{G})=\mathrm{Z} \_\mathrm{v}$. So, the automorphism can be represented by

$a: i \rightarrow i+1(" m o d " v)$ or $a:(0,1, \cdots, v-1)$

A starter set of a cyclic $(\mathrm{G}, \mathrm{Y})$-design is a set of subgraphs of $G$ that generates all subgraphs of $Y$ by repeated addition of 1 modular $\mathrm{v}$.

A complete multigraph of order $\mathrm{v}$, denoted by $\lambda \mathrm{K} \_\mathrm{v}$, is obtained by replacing each edge of $K_{-} v$ with $\lambda$ edges. The problem which concerned in the decomposition of the complete multigraph into subgraphs has received much attention in recent years. The necessary and sufficient conditions for decomposing $\lambda \mathrm{K} v$ into cycles of order $\lambda$ and cycles of prime oredr have been established by [2]. While, the existence theorem of m-cycle system of $\lambda \mathrm{K}_{-} \mathrm{v}$ has been proved for all values of $\lambda$ in [3]. For the important case of $\lambda=1$, the existence question for m-cycle system of order $v$ has been completely settled by [4] in the case m odd and by [5] in the case $m$ even. Moreover, the cyclic m-cycle system of order $\mathrm{v}$ for $\mathrm{m}=3$, denoted by $\operatorname{CTS}(\mathrm{v}, \lambda)$, has been constructed by [6] and for a cyclic Hamiltonian cycle system of order $\mathrm{v}$ was proved when $\mathrm{v}$ is an odd integer but $\mathrm{v} \neq 15$ and $\mathrm{v} \neq \mathrm{p}^{\wedge} \alpha$ with $\mathrm{p}$ a prime and $\alpha>1$ [7].

On the other hand, the necessary and sufficient conditions for decomposing $\lambda \mathrm{K}_{-} \mathrm{v}$ into cycle and star graphs have been investigated by [8].

A four-factor of a graph $\mathrm{G}$ is a spanning subgraph whose vertices have a degree 4 . While a near-four-factor is a spanning subgraph in which all vertices have a degree four with exception of one vertex (isolated vertex) which has a degree zero [9].

In this paper, we propose a new type of cyclic cycle system that is called cyclic near Hamiltonian cycle system of $4 \mathrm{~K} \_\mathrm{v}$, denoted CNHC(4K_v,C_(v-1) ). This is obtained by combination a near-four-factors and cyclic ( $\mathrm{v}-1)$-cycle system of $4 \mathrm{~K} \_\mathrm{v}$ when $\mathrm{v} \equiv 2(\bmod 4)$. Furthermore, the construction of $\mathrm{CNHC}\left(4 \mathrm{~K} \_\mathrm{v}, \mathrm{C} \_(\mathrm{v}-1)\right)$ will be employed to decompose $8 \mathrm{~K} \_\mathrm{v}$ into Hamiltonian wheels.

\section{PRELIMINARIES}

In our paper, all graphs considered have vertices in $Z_{V}$. We will use the difference set method to construct the desired designs. The difference between any two distinct vertices $a$ and $b$ in $\lambda K_{\mathrm{V}}$ is $\pm|a-b|$, arithmetic (mod v). Given $C_{m}=\left(c_{0, \ldots,}, c_{m-1}\right)$ an $m$-cycle, the differences from 
$C_{m}$ are the multiset $\Delta\left(C_{m}\right)=\left\{ \pm\left|c_{i}-c_{i-1}\right| \mid i=1,2, \ldots, m\right\}$ where $c_{0}=c_{m}$. Let $\mathcal{F}=\left\{B_{1}, B_{2, \ldots,} B_{\gamma}\right\}$ be an $m$-cycles of $\lambda K_{V}$ the list of differences from $\mathcal{F}$ is $\Delta(\mathcal{F})=\bigcup_{i=1}^{F} \Delta\left(B_{i}\right)$.

The orbit of cycle $C_{m}$, denoted by orb $\left(C_{m}\right)$, is the set of all distinct $m$-cycles in the collection $\left\{C_{m}+i \mid i \in Z_{V}\right\}$. The length of $\operatorname{arb}\left(C_{m}\right)$ is its cardinality, i.e., $\operatorname{arb}\left(C_{m}\right)=k$ where $k$ is the minimum positive integer such that $C_{m}+k=C_{m}$. A cycle orbit of length $v$ on $\lambda K_{V}$ is said full and otherwise short. [10]

The stabilizer of a subgraph $H$ of a graph $G$ of order $v$ is $\operatorname{stab}(H)=\left\{z \in Z_{D} \mid z+H=H\right\}$ and $H$ has trivial stabilizer when $\operatorname{stab}(H)=\{0\}$. One may easily deduce the following result.

For presenting a cyclic $m$-cycle system of $\lambda K_{W}$, it sufficient to construct a starter set, i.e., $m$-cycle system of representations for its cycle orbits. As particular consequences of the theory developed in [11] we have:

Lemma 1. Let $H$ be a subgraph of $G$ and $|\operatorname{stab}(H)|>1$. Then each nonzero integer in $\Delta H$ appears a multiple of |stab $(H) \mid$ times.

Lemma 2. Let $\delta$ be a multiset of subgraphs of $\lambda K_{\mathrm{V}}$ and every subgraph of $\delta$ has trivial stabilizer. Then $\delta$ is a starter of cyclic $\left(\lambda K_{W^{\prime}} y\right)$-design if and only if $\Delta \delta$ covers each nonzero integer of $Z_{V}$ exactly $\lambda$ times.

\section{CYCLIC NEAR HAMILTONIAN CYCLE SySTEM}

Definition 1. A full cyclic near Hamiltonian cycle system of the $4 K_{\mathrm{V}}$, denoted by $\operatorname{CNHC}\left(4 K_{\mathrm{W}} C_{\mathrm{V}-1}\right)$, is a cyclic $(v-1)$-cycle system of $4 K_{\mathrm{V}}$ graph, that satisfies the following conditions:

1. The cycle in row $r$ form a near-4-factor with focus $r$.

2. The cycles associated with the rows contain no repetitions.

Surely, for presenting a full cyclic near Hamiltonian cycle system of the $4 K_{\mathrm{V}}, \mathrm{CNHC}\left(4 K_{\mathrm{W}^{x}} C_{\mathrm{V}-1}\right)$, it is sufficient to provide a set of starter set that satisfies a near-4-factor. We give here example to explain the above definition.

Example 1. Let $G=4 K_{14}$ and $\mathcal{F}=\left\{C_{13}, C_{13}^{*}\right\}$ is a set of 13 -cycles of $G$ such that:

$$
\begin{aligned}
& C_{13}=(1,13,2,12,3,11,4,5,10,6,9,7,8), \\
& C_{13}^{*}=(13,8,12,9,11,10,4,3,5,2,6,1,7) .
\end{aligned}
$$

Firstly, it is easy to observe that each non zero element in $Z_{14}$ occurs exactly twice in the 13 -cycles of $\mathcal{F}$. Since, the cycle graph is 2-regular graph, then every vertex has a degree 4 except a zero element (isolated vertex) has a degree zero. Thus, it is satisfies the near-4-factor with focus zero element. Secondly, the list of differences set of the set $\mathcal{F}$ is listed in Table I

TABLE I

THE LIST OF DIFFERENCES OF $\mathcal{F}$

\begin{tabular}{|c|c|}
\hline 13-cycles & \multicolumn{1}{c|}{ Difference set } \\
\hline$(1,13,2,12,3,11,4,5,10,6,9,7,8)$ & $\left\{\begin{array}{c}2,12,3,11,4,10,5,9,6,8,7,7,1,13, \\
5,9,4 ; 10,3,11,2,12,1,13,7,7\end{array}\right]$ \\
\hline$(13,8,12,9,11,10,4,3,5,2,6,1,7)$ & $\left\{\begin{array}{c}5,9,4,10,3,11,2,12,1,13,6,8,1,13 \\
2,12,3,11,4,10,5,9,6,8,6,8\end{array}\right]$ \\
\hline
\end{tabular}

It can be seen from the Table I, $\Delta(\mathcal{F})=\Delta\left(C_{13}\right) \cup \Delta\left(C_{13}^{*}\right)$ covers each nonzero element in $Z_{14}$ exactly four times. Since the cycles set $\mathcal{F}$ has trivial stabilizer based on Lemma 1 , then the set $\mathcal{F}=\left\{C_{13}, C_{13}^{*}\right\}$ is the starter set of $\operatorname{CNHC}\left(4 K_{14}, C_{13}\right)$ by Lemma 2 .

Therefore, $\mathrm{CNHC}\left(4 K_{14}, C_{18}\right)$ is an $(14 \times 2)$ array design and cycles set $\mathcal{F}=\left\{C_{13}, C_{19}^{b}\right\}$ in the first row generates all cycles in $(14 \times 2)$ array by repeated addition of 1 modular 14 as shown in the Table II.

TABLE II

$C N H C\left(4 K_{14} C_{99}\right)$

\begin{tabular}{|l|l|l|}
\hline \multicolumn{1}{|c|}{ Focus } & \multicolumn{2}{|c|}{ CNHC $\left(4 K_{14}, C_{13}\right)$} \\
\hline$r=0$ & $(1,13,2,12,3,11,4,5,10,6,9,7,8)$ & $(13,8,12,9,11,10,4,3,5,2,6,1,7)$ \\
\hline$r=1$ & $(2,0,3,13,4,12,5,6,11,7,10,8,9)$ & $(0,9,13,10,12,11,5,4,6,3,7,2,8)$ \\
\hline$r=2$ & $(3,1,4,0,5,13,6,7,12,8,11,9,10)$ & $(1,10,0,11,13,12,6,5,7,4,8,3,9)$ \\
\hline$\geqq$ & $\geqq$ & $\geqq$ \\
\hline$r=13$ & $(0,12,1,1,2,10,3,4,9,5,8,6,7)$ & $(12,7,11,8,10,9,3,2,4,1,5,0,6)$ \\
\hline
\end{tabular}

Throughout the paper, a near Hamiltonian cycle of order $(v-1)$ will be represented as connected paths, we mean

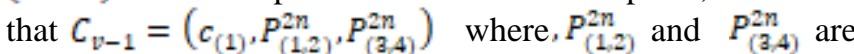
(2n)-paths such that:

$$
\begin{aligned}
& P_{[1,2)}^{2 n}=\left[c_{(1,1),} c_{(2,1)}, c_{(1,2),}, c_{(2,2), \ldots,}, c_{[1, n\}}, c_{(2, n)}\right], \\
& =\left[\bigcup_{i=1}^{n} c_{(1, i),}^{n} c_{(2, i)}\right]
\end{aligned}
$$

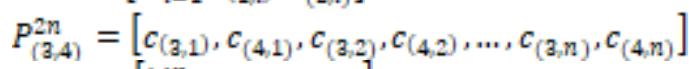

$$
\begin{aligned}
& =\left[\bigcup_{i=1}^{n} c_{(a, i)}^{n} c_{(4, i)}\right] \text {. }
\end{aligned}
$$

Let the vertex sets of $P_{[1,2\}}^{2 n}$ and $P_{[3,4]}^{2 n}$ are

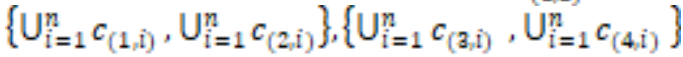
respectively. And the list of difference sets of $P_{[1,2)}^{2 n}$ and $P_{[(\pi, 4]}^{2 n}$ will be calculated as follows:

$$
\begin{aligned}
& \Delta\left(P_{[1,2]}^{2 n}\right)=\Delta_{1}\left(P_{[1,2]}^{2 n}\right) \cup \Delta_{2}\left(P_{[1,2]}^{2 n}\right), \\
& \Delta\left(P_{[3,4]}^{2 n}\right)=\Delta_{1}\left(P_{[\{, 4]}^{2 n}\right) \cup \Delta_{2}\left(P_{[3,4]}^{2 n}\right) \text { such that } \\
& \Delta_{1}\left(P_{[1,2)}^{2 n}\right)=\left\{ \pm\left|c_{[1, i)}-c_{(2, i)}\right| \mid 1 \leq i \leq n\right\} \text {. } \\
& \Delta_{2}\left(P_{[1,2]}^{2 n}\right)=\left\{ \pm\left|c_{[1, i+1)}-c_{[2, n\}}\right| \mid 1 \leq i \leq n-1\right\} \text {. } \\
& \Delta_{1}\left(P_{[a, 4\}}^{2 n}\right)=\left\{ \pm\left|c_{[a, i\}}-c_{\{4, i\})}\right| 1 \leq i \leq n\right\} \text {. } \\
& \Delta_{2}\left(P_{[a, 4]}^{2 n}\right)=\left\{ \pm \mid c_{[a, i+1)}-c_{[4, i]} \| 1 \leq i \leq n-1\right\} \text {. }
\end{aligned}
$$


And we define $\Delta\left(c_{[1)^{n}} P_{[1,2]}^{2 n}\right), \Delta\left(P_{\left[\{, 4\}^{n}\right.}^{2 n} c_{\{1\}}\right)$ and $\Delta\left(P_{[1,2],}^{2 n}, P_{[a, 4]}^{2 n}\right)$ as follows

$\Delta\left(c_{[1)^{x}} P_{[1,2]}^{2 n}\right)= \pm\left|c_{(1)}-c_{[1,1\}}\right|$,

$\Delta\left(P_{[3,4]}^{2 n}, c_{[1]}\right)= \pm\left|c_{[4, n]}-c_{(\mathbb{1})}\right|$

$\Delta\left(P_{[1,2]}^{2 n}, P_{[\{, 4]}^{2 n}\right)= \pm\left|c_{[2, n\}}-c_{[3,1]}\right|$.

So, the list of difference of $C_{\mathrm{V}-1}$ shall be represented as a follows:

$\Delta\left(C_{V-1}\right)=\Delta\left(P_{[1,2]}^{2 n}\right) \cup \Delta\left(P_{[\mathbb{2}, 4]}^{2 n}\right) \cup \Delta\left(c_{[1)^{n}} P_{[1,2]}^{2 n}\right) \cup$

$\Delta\left(P_{[a, 4\}^{2}}^{2 n}, c_{[1]}\right) \cup \Delta\left(P_{[1,2]}^{2 n}, P_{[\{, 4\}}^{2 n}\right)$

Now we are able to provide our main result.

Theorem 1. There exists a full cyclic near Hamiltonian cycle system of $4 K_{\mathrm{V}}, \operatorname{CNHC}\left(4 K_{\mathrm{W}^{s}} C_{\mathrm{V}-1}\right)$, when $v=4 n+2, n>2$.

Proof. Suppose $\mathcal{F}=\left\{C_{4 n+1}, C_{4 n+1}^{*}\right\}$ is a set of near Hamiltonian cycles of $4 K_{4 n+2}$ where

$$
\begin{aligned}
& C_{4 n+1}=\left(1_{s} P_{[1,2]]^{n}}^{2 n} P_{[3,4]}^{2 n}\right), \\
& C_{4 n+1}^{*}=\left(2 n+1, P_{[1,2]}^{\left([2 n)^{\infty}\right.}, P_{[(3,4)}^{(2 n)^{\infty}}\right)
\end{aligned}
$$

Such that:

- $P_{[1,2]}^{2 n}=[4 n+1,2,4 n, 3, \ldots, 3 n+2, n+1]$.

$=\left[\bigcup_{i=1}^{n} 4 n+2-i, i+1\right]$

$P_{(3,4)}^{2 n}=[n+2,3 n+1, n+3,3 n, \ldots, 2 n+1,2 n+$

- 2$]==\left[\bigcup_{i=1}^{n} n+1+i_{v} 3 n+2-i\right]$

$$
P_{(1,2)}^{(2 n)^{\infty}}=[4 n+1,2 n+2,4 n, 2 n+3, \ldots, 3 n+
$$

- $2,3 n+1]=\left[\bigcup_{i=1}^{n} 4 n+2-i_{s} 2 n+1+i\right]$

- $\dot{P}_{(\mathrm{g}, 4)}^{(\mathrm{d} n)^{\infty}}=[n+1, n, n+2, n-1, \ldots, 2 n, 1]$

$$
=\left[\bigcup_{i=1}^{n} n+i_{s} n+1-i\right] \text {. }
$$

We will divide the proof into two parts as follows:

Part 1. In this part will be proved that $\mathcal{F}$ satisfies a near-4factor. We shall calculate the vertex set of $C_{4 n+1}$ and $C_{4 n+1}^{*}$ such that:

$V\left(C_{4 n+1}\right)=V\left(P_{[1,2]}^{2 n}\right) \cup V\left(P_{[3,4}^{2 n}\right) \cup\{1\}$.

$V\left(C_{4 n+1}^{*}\right)=V\left(P_{[(1,2)}^{(2 n)^{*}}\right) \cup V\left(P_{(2,4)}^{(2 n)^{*}}\right) \cup\{2 n+1\}$.

$\bigcup_{i=1}^{n} c_{[1, i}=\{4 n+2-i, 1 \leq i \leq n\}$

$$
=\left\{4 n+1,4 n_{v \ldots,}, 3 n+2\right\} \text {, }
$$

$\mathrm{U}_{i=1}^{n} c_{\{2,1}=\{i+1,1 \leq i \leq n\}=\{2,3, \ldots, n+1\}$,

$\bigcup_{i=1}^{n} c_{[a i 1}=\{n+1+i, 1 \leq i \leq n\}$

$$
=\{n+2, n+3, \ldots, 2 n+1\},
$$

$\mathrm{U}_{i=1}^{n} c_{4, i 1}=\{3 n+2-i, \quad 1 \leq i \leq n\}$

$$
=\{3 n+1,3 n, \ldots, 2 n+2\} .
$$

From above equations, it is easy to notice that $V\left(C_{4 n+1}\right)$ covers each nonzero element of $Z_{4 n+2}$ exactly once.

$$
\begin{aligned}
\bigcup_{i=1}^{n} c_{[1, i n}^{*} & =\{4 n+2-i, 1 \leq i \leq n\} \\
& =\{4 n+1,4 n, \ldots, 3 n+2\}, \\
\bigcup_{i=1}^{n} c_{[2, i)}^{*} & =\{2 n+1+i, 1 \leq i \leq n\} \\
& =\{2 n+2,2 n+3, \ldots, 3 n+1\}, \\
\bigcup_{i=1}^{n} c_{[3, i]}^{*} & =\{n+i, 1 \leq i \leq n\} \\
& =\{n+1, n+2, \ldots, 2 n\}
\end{aligned}
$$

It can be observed from the above equations that $V\left(C_{4 n+1}\right)=V\left(C_{4 n+1}^{*}\right)$. Then, the multiset $V\left(C_{4 n+1}\right) \cup V\left(C_{4 n+1}^{*}\right)$ covers each nonzero elements of $Z_{4 n+2}$ exactly twice. Since the cycle graph is 2 -regular graph, therefore $\mathcal{F}=\left\{C_{4 n+1}, C_{4 n+1}^{*}\right\}$ satisfies a near-fourfactor (with focus zero).

Part 2. In this part we will prove $\mathcal{F}=\left\{C_{4 n+1}, C_{4 n+1}^{*}\right\}$ is the starter set of cyclic $(v-1)$-cycle system of $4 K_{V}$. So, we will calculate the difference set of each of them as follows:

$$
\begin{aligned}
& \Delta\left(C_{4 n+1}\right)=\Delta\left(P_{[1,2]}^{2 n}\right) \cup \Delta\left(P_{[\mathbb{2}, 4)}^{2 n}\right) \cup \Delta\left(c_{[\mathbb{1}]}, P_{[1,2]}^{2 n}\right) \cup \\
& \Delta\left(P_{(1,2)}^{2 n}, P_{(1,4)}^{2 n}\right) \cup \Delta\left(P_{(3,4)}^{2 n}, c_{(1)}\right)
\end{aligned}
$$

$\Delta_{1}\left(P_{[1,2)}^{2 n}\right)=U_{i=1}^{n} \pm|4 n+1-2 i|=\{4 n-1,4 n-$

- $3, \ldots, 2 n+1\} \cup\{3,5, \ldots, 2 n+1\}$

$$
\Delta_{2}\left(P_{[1,2)}^{2 n}\right)=\bigcup_{i=1}^{n n-1} \pm|4 n-2 i|=\{4 n-2,4 n-
$$

- $4, \ldots, 2 n+2\} \cup\{4,6, \ldots, 2 n\}$

$$
\dot{\Delta}_{1}\left(P_{[, 4}^{2 n}\right)=U_{i=1}^{n} \pm|2 n+1-2 i|=\{2 n-1,2 n-
$$

- $3, \ldots, 1\} \cup\{2 n+3,2 n+5, \ldots, 4 n+1\}$

$$
\Delta_{2}\left(P_{[a, 4)}^{2 n}\right)=U_{i=1}^{n-1} \pm|2 n-2 i|=\{2 n-2,2 n-
$$

- $\left.4{ }_{s, z} 2\right\} \cup\{2 n+4,2 n+6, \ldots, 4 n\}$

- $\Delta\left(c_{[1],}, P_{[1,2]}^{2 n}\right)= \pm\left|c_{[1]}-c_{[\mathbb{1}, \mathbb{1}}\right|=\{2,4 n\}$,

- $\Delta\left(P_{[1,2]}^{2 n}, P_{(3,4)}^{2 n}\right)= \pm\left|c_{[2, n)}-c_{[(3,1)}\right|=\{1,4 n+1\}$.

- $\Delta\left(P_{[\{, 4\}}^{2 n}, c_{[1,1)}\right)= \pm\left|c_{[4, n}-c_{[\mathbb{1}\}}\right|=\{2 n+1,2 n+1\}$

From the equation 9, We note that the list of differences of $C_{4 n+1}, \Delta\left(C_{4 n+1}\right)$, covers each nonzero elements of $Z_{4 n+2}$ twice except the differences $\{2 n, 2 n+2\}$ appear once.

Now we will calculate $\Delta\left(C_{4 n+1}^{*}\right)$ such as

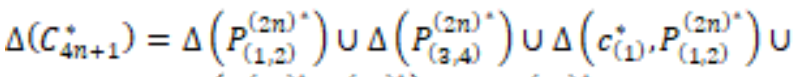

$$
\begin{aligned}
& \Delta\left(P_{(1,2)}^{(2 n)}, P_{(3,4)}^{(2 n)}\right) \cup \Delta\left(P_{(3,4)}^{(2 n)^{2}}, c_{[1]}^{*}\right)
\end{aligned}
$$

$$
\Delta_{1}\left(P_{(1,2)}^{(2 n)^{\infty}}\right)=\bigcup_{i=1}^{n} \pm|2 n+1-2 i|=\{2 n-
$$

- $1,2 n-3, \ldots, 1\} \cup\{2 n+3,2 n+5, \ldots, 4 n+1\}$ 
$\Delta_{2}\left(P_{[1,2]}^{(2 n)^{2}}\right)=U_{i=1}^{n-1} \pm|2 n-2 i|=\{2 n-2,2 n-$

- $4, \ldots, 2\} \cup\{2 n+4,2 n+6, \ldots, 4 n\}$

$$
\Delta_{1}\left(P_{[a, 4)}^{(2 n)^{\circ}}\right)=U_{i=1}^{n} \pm|2 i-1|=\{1,3, \ldots, 2 n-1\} \cup
$$

- $\{4 n+1,4 n-1, \ldots, 2 n+3\}$

$$
\Delta_{2}\left(P_{(2,4)}^{(2 n)^{*}}\right)=\bigcup_{i=1}^{n-1} \pm|2 i|=\{2,4, \ldots, 2 n-2\} \cup
$$

- $\{4 n, 4 n-2, \ldots, 2 n+4\}$

- $\Delta\left(c_{[1,}^{*}, P_{[1,2]}^{[(2 n)}\right)= \pm\left|c_{[\mathbb{1} 1}^{*}-c_{[1,1]}^{*}\right|=\{2 n, 2 n+2\}$. $\Delta\left(P_{[1,2\}}^{(2 n)^{*}}, P_{(2,4)}^{(2 n)^{*}}\right)= \pm\left|c_{[2, n)}^{*}-c_{[3,1}^{*}\right|=\{2 n, 2 n+$

- 23

- $\Delta\left(P_{[(2,4)}^{(2 n)^{\infty}}, c_{[\mathbb{1}}^{*}\right)= \pm\left|c_{[4, n)}^{*}-c_{[\mathbb{1}}^{*}\right|=\{2 n, 2 n+2\}$.

As clearly shown, in the equations 10 , every nonzero element in $Z_{4 n+2}$ appears twice except $\{2 n, 2 n+2\}$ appear three times in $\Delta\left(C_{4 n+1}^{*}\right)$. Based on Lemma 1, the cycles $\left\{C_{4 n+1}, C_{4 n+1}^{*}\right\}$ have trivial stabilizer.

One can easily note that $\Delta(\mathcal{F})=\Delta\left(C_{4 n+1}\right) \cup \Delta\left(C_{4 n+1}^{*}\right)$ covers each non zero integers in $Z_{4 n+2}$ four times. Thus, $\mathcal{F}=\left\{C_{4 n+1}, C_{4 n+1}^{*}\right\}$ is the starter cycles of cyclic $(v-1)$ cycle system of $4 K_{v}$ by Lemma 2 . Hence, the cycles set $\mathcal{F}=\left\{C_{4 n+1}, C_{4 n+1}^{*}\right\}$ generates a full near Hamiltonian cycle system of $4 \mathrm{~K}_{\mathrm{v}}$ by adding one modular $v$ when $v=4 n+2, n>2$

\section{CYCLIC HAMILTONIAN WHEEL SYSTEM}

A wheel graph of order $m$, denoted by $W_{m}$, consists of a singleton graph $K_{1}$ and a cycle graph of order $m-1, C_{m-1}$, in which the $K_{1}$ is connected to all the vertices of $C_{m-1}$, written $K_{1}+C_{m-1}$ or $c_{0}+\left(c_{1}, c_{2}, \ldots, c_{m-1}\right)$. An $m$-wheel contains $2(m-1)$ edges such that the edge set of $W_{m}$ is $E\left(W_{m}\right)=E\left(K_{1,(m-1)}\right) \cup E\left(C_{m-1}\right)[12]$.

An $m$-wheel system of graph $G$ is a decomposition of edge set of $G$ into collection $W=\left\{W_{m_{1}, \ldots \ldots,} W_{m_{\mathrm{r}}}\right\}$ of edgesdisjoint of $m$-wheels. Similar to the cyclic cycle system, an $m$-wheel system of $\lambda K_{V}$ is a cyclic if $V\left(\lambda K_{V}\right)=Z_{V}$ and if $W_{m}=c_{0}+\left(c_{1}, c_{2}, \ldots, c_{m-1}\right) \in W \quad$ implies that $W_{m}+1=\left(c_{0}+1\right)+\left(c_{1}+1, c_{2}+1, \ldots, c_{m-1}+1\right)$ is also in $w$. Moreover, if $m=v$ then it is called a cyclic Hamiltonian wheel system. The list of difference of $W_{m}=c_{0}+\left(c_{1}, c_{2}, \ldots, c_{m-1}\right)$

$\Delta\left(W_{m}\right)=\Delta\left(K_{1 / m-1}\right) \cup \Delta\left(C_{m-1}\right) \quad$ such that $\Delta\left(c_{m}\right)=\left\{ \pm \mid c_{i+1}-c_{i} \| 1 \leq i \leq m-1\right\}$ where $c_{m}=c_{0}$ and $\Delta\left(K_{[1,(m-1)]}\right)=\left\{ \pm\left|c_{i}-c_{0}\right| 11 \leq i \leq m-1\right\}$. More generally, given a multiset $W=\left\{W_{m M_{1}, \ldots,}, W_{m_{p}}\right\}$ of $m$ wheels of $\lambda K_{W}$, the list of differences from $W$ is $\Delta W=\mathrm{U}_{i=1}^{t} \Delta W_{m_{\mathrm{i}}}$.

Definition 2. The full cyclic Hamiltonian wheel system of a graph $8 K_{V}$, denoted by $C H W S\left(8 K_{V}, W\right)$, is a cyclic (v)wheel system of a graph $8 K_{V}$ that generated by starter set $W=\left\{W_{V_{1}, \ldots,}, W_{V_{p}}\right\}$ such that the associated cycles with wheels satisfy near-four-factor with focus singleton graph.
In other words CHWS $\left(8 K_{v}, W\right)$ is a $(v \times|w|)$ array such that satisfies the following conditions:

1) The wheels in row $r$ form a $r+$ (near-four-factor).

2) The wheels associated with the rows contain no repetitions.

For clarity, we provide an example to demonstrate the construction of a cyclic Hamiltonian wheel system stated above.

Example 2. Let $G=8 K_{14}$ and $W=\left\{W_{14}, W_{14}^{*}\right\}$ is a set of Hamiltonian wheels of $8 K_{14}$. Where

$W_{14}=K_{1}+C_{13}=0+$

$(1,13,2,12,3,11,4,5,10,6,9,7,8)$

and

$W_{14}^{*}=K_{1}^{*}+C_{13}^{*}=0+(13,8,12,9,11,10,4,3,5,2,6,1,7)$

From the Example 1, we can note that the 13-cycles $\left\{C_{13}, C_{13}^{*}\right\}$ satisfy a near-four-factor with focus 0 (zero element). Moreover, the list of differences from $\left\{C_{13}, C_{13}^{*}\right\}$ covers every nonzero element of $Z_{14}$ exactly four times. Now we want to find the list of differences from $\left(K_{1,133)}\right) \cup\left(K_{1,(13)}^{*}\right)$ as a follows

$$
\begin{aligned}
& \Delta\left(K_{1 / 13}\right)=\left\{ \pm\left|c_{i}-c_{0}\right| 11 \leq i \leq 13\right\} \\
& =\left\{ \pm\left|c_{i}\right| \quad \text { I1 } \leq i \leq 13\right\}
\end{aligned}
$$

such that the $c_{\mathrm{i}} \in V\left(C_{13}\right), 1 \leq i \leq 13$. Since the vertex set of $C_{13}$ is $Z_{14}-\{0\}$ and $Z_{14}=-Z_{14}$, then $\Delta\left(K_{1,(13)}\right)$ covers each nonzero element of $Z_{14}$ twice. Similarly, one can be found $\Delta\left(K_{1(13)}^{*}\right)=\Delta\left(K_{1,(13)}\right)$. So $\Delta(w)$ covers each nonzero of $Z_{14}$ four times. Thus, $W=\left\{W_{14}, W_{14}^{*}\right\}$ is the starter set of $C H W S\left(8 K_{14}, W\right)$.

Then $C H W S\left(8 K_{14}, W_{14}\right)$ is an $(14 \times 2)$ array deign where all its wheels can be generated by repeated addition 1

\begin{tabular}{|c|c|}
\hline \multicolumn{2}{|c|}{ CHWS $\left(8 K_{14}, W\right)$} \\
\hline $0+(1,13,2,12,3,11,4,5,10,6,9,7,8)$ & $0+(13,8,12,9,11,10,4,3,5,2,6,1,7)$ \\
\hline $1+(2,0,3,13,4,12,5,6,11,7,10,8,9)$ & $1+(0,9,13,10,12,11,5,4,6,3,7,2,8)$ \\
\hline $2+(3,1,4,0,5,13,6,7,12,8,11,9,10)$ & $2+(1,10,0,11,13,12,6,5,7,4,8,3,9)$ \\
\hline 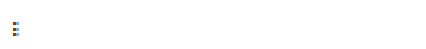 & $\geqq$ \\
\hline $13+(0,12,1,1,2,10,3,4,9,5,8,6,7)$ & $13+(12,7,11,8,10,9,3,2,4,1,5,0,6)$ \\
\hline
\end{tabular}
(modular 14) on the starter set $W$ as shown in the Table III.

TABLE III

CHWS $\left(8 K_{14} W_{14}\right)$

The following theorem proves the existence of CHWS $\left(8 K_{4 m+2}, W\right)$.

Theorem 2. There exists a full cyclic Hamiltonian wheel system of $8 K_{v}, C H W S\left(8 K_{V}, w\right)$, for $v=4 n+2, n>2$.

Proof. We have to present a starter set $W=\left\{K_{1}+C_{4 n+1}, K_{1}^{*}+C_{4 n+1}^{*}\right\}$ of CHWS $\left(8 K_{\mathrm{V},}, w\right)$ such 
that the cycles associated with the wheels in $W$ satisfy a near-four-factor with focus a singleton graph.

Suppose $W=\left\{0+C_{4 n+1}, 0+C_{4 n+1}^{*}\right\}$ is a set of Hamiltonian wheels of $8 K_{4 n+2}$ where

$$
\begin{aligned}
& C_{4 n+1}=\left(1, P_{[1,2]^{n}}^{2 n} P_{[(2,4]}^{2 n}\right), \\
& C_{4 n+1}^{*}=\left(2 n+1, P_{[1,2)}^{(2 n)^{\infty}}, P_{[(2,4)}^{(2 n)^{\infty}}\right)
\end{aligned}
$$

Such that:

- $\quad P_{[1,2]}^{2 n}=[4 n+1,2,4 n, 3, \ldots, 3 n+2, n+1]$.

$$
P_{[(3,4)}^{2 n}=\left[n+2,3 n+1, n+3,3 n_{, \ldots,} 2 n+1,2 n+\right.
$$

- 2]

$$
P_{(1,2)}^{(2 n)^{\infty}}=[4 n+1,2 n+2,4 n, 2 n+3, \ldots, 3 n+
$$

- $2,3 n+1]$

- $P_{[2,4]}^{\left[(2 n)^{\infty}\right.}=\left[n+1, n, n+2, n-1_{, \ldots,}, 2 n, 1\right]$.

From Theorem 1, the cycles associated with the Hamiltonian wheels in $W$ satisfy the near-four-factor with focus zero element.

Now, we want to prove $W=\left\{K_{1}+C_{4 n+1} K_{1}^{*}+C_{4 n+1}^{*}\right\}$ is a $\left(\lambda K_{\mathrm{p}}, \mathcal{W}\right)$-difference system. To do this, it is enough to show that the list of differences

$$
\begin{gathered}
\Delta W=\left\{\Delta\left(C_{4 n+1}\right) \cup \Delta\left(C_{4 n+1}^{*}\right) \cup \Delta\left(K_{1 / 4 n+1}^{*}\right) \cup\right. \\
\left.\Delta\left(K_{1,[4 n+1)}\right)\right\}
\end{gathered}
$$

covers each element of $\left\{Z_{4 n+2}-\{0\}\right\}$ eight times. Firstly, as indicated in Theorem 1 , the list of differences of $\left\{\left(C_{4 n+1}\right) \cup\left(C_{4 n+1}^{*}\right)\right\}$ cover each nonzero element in $Z_{4 n+2}$ exactly four times.

Secondly, the list of differences of $\left(K_{1 / 4 n+1)}\right)$ is $\left\{ \pm\left|c_{\mathfrak{i}}-0\right| \| c_{i} \in C_{4 n+1}\right\}$. Since $V\left(C_{4 n+1}\right)=Z_{4 n+2}-\{0\}$ then $\left\{c_{i}-0 \mid \| c_{i} \in C_{4 n+1}\right\}=Z_{4 n+2}-\{0\}$. Because of $Z_{4 n+2}=-\left\{Z_{4 n+2}\right\} \quad$, then $\Delta\left(K_{1 / 4 n+1)}\right)=\left\{ \pm\left|c_{i}-0\right| \| c_{i} \in C_{4 n+1}\right\} \quad$ covers each nonzero element of $Z_{4 n+2}$ twice. Likewise, we repeat the same strategy on cycle $K_{1 / 4 n+1)}^{*}$ to find $\Delta\left(K_{1 /(4 n+1)}^{*}\right)$. Also, it is an easy matter to check that $\Delta\left(K_{1 / 4 n+1)}^{*}\right)=\Delta\left(K_{1(4 n+1)}\right)$.

Linking together the above list of differences, we see that $\Delta W$ covers each nonzero element of $Z_{4 n+2}$ eight times. On the other hand, each wheel graph in $W$ has trivial stabilizer based on Lemma 1 . Therefore, $W$ is the starter set of
CHWS $\left(8 K_{\mathrm{V}}, W\right)$, by Lemma 2 . One can be generated CHWS $\left(8 K_{\mathrm{V},}, W\right)$ by repeated addition 1 modular $v$ on $W$.

\section{CONCLUSION}

In this paper, we have provided new designs $\operatorname{CNHC}\left(4 K_{\mathrm{W},} C_{\mathrm{V}-1}\right)$ and $\operatorname{CHWS}\left(8 K_{\mathrm{V}}, W_{\mathrm{V}}\right)$ where $\nu \equiv 2$ (mod4) . These designs are interested in a decomposition of complete multigraph into cyclic $(v-1)$. cycle and cyclic (v) -wheel graphs, respectively. We have also proved the existence of these designs by constructed the starter set for each of them. Moreover, one can ask if $\operatorname{CNHC}\left(2 \lambda K_{\mathrm{V}} C_{\mathrm{V}-1}\right)$ and $\operatorname{CHWS}\left(2 \lambda K_{\mathrm{W}}, W_{\mathrm{p}}\right)$ can be constructed for the case $v \equiv 2,4(\bmod 4)$ and $\lambda>2$

\section{REFERENCES}

[1] S. L. Wu and H. C. Lu, "Cyclically decomposing the complete graph into cycles with pendent edges". ARS COMBINATORIAWATERLOO THEN WINNIPEG, vol. 86, no. 217, 2008.

[2] B. R. Smith, "Cycle decompositions of complete multigraphs," Journal of Combinatorial Designs, vol. 18, no. 2, pp.85-93, 2010.

[3] D. Bryant, D. Horsley, B. Maenhaut and B. R. Smith, "Cycle decompositions of complete multigraphs", Journal of Combinatorial Designs, vol. 19, no. 1, pp. 42-69, 2011.

[4] B. Alspach and H. Gavlas, "Cycle decompositions of K n and K_nI,”. Journal of Combinatorial Theory, Series B, vol. 81, no. 1, pp. 77 99, 2001.

[5] M. Šajna, "Cycle decompositions III: complete graphs and fixed length cycles," Journal of Combinatorial Designs, vol. 10, no. 1, pp. 27-78, 2002.

[6] M. J. Colbourn and C. J. Colbourn, "Cyclic block designs with block size 3," European Journal of Combinatorics, vol, 2, no. 1, pp. 21-26, 1981.

[7] M. Buratti and A. Del Fra, "Cyclic Hamiltonian cycle systems of the complete graph," Discrete mathematics, vol. 279, no. 1, pp. 107-119, 2004 .

[8] F. Beggas, M. Haddad and H. Kheddouci, "Decomposition of Complete Multigraphs Into Stars and Cycles," Discussiones Mathematicae Graph Theory, vol. 35, no. 4, pp. 629-639, 2015.

[9] R. S. Rees, "The spectrum of triangle-free regular graphs containing a cut vertex," Australasian Journal of Combinatorics, vol. 26, pp. 135-14, 2002

[10] S. L. Wu and H. L. Fu, "Cyclic $m$ - cycle systems with $m \leqslant 32$ or $\mathrm{m}=2 \mathrm{q}$ with q a prime power,” . Journal of Combinatorial Designs, vol. 14 , no. 1 , pp. $66-81,2006$

[11] M. Buratti, "A description of any regular or 1-rotational design by difference methods," Booklet of the abstracts of Combinatorics, pp. 35-52, 2000.

[12] S. Pemmaraju and S. Skiena, "Cycles, stars, and wheels," Computational Discrete Mathematics Combinatiorics and Graph Theory in Mathematica, pp. 284-249, 2003. 